\title{
AN INFINITE CLASS OF IRREDUCIBLE HOMOLOGY 3-SPHERES
}

\author{
JONATHAN L. GROSS
}

\begin{abstract}
A class of irreducible homology 3-spheres is obtained by pasting together complements of torus knots. Representations of the fundamental groups of these homology 3-spheres into symmetric groups are then used to distinguish the members of an infinite subclass.
\end{abstract}

In this paper, a knot complement means the complement in a 3 -sphere of the interior of a regular neighborhood of an imbedded 1 -sphere. The objects of concern are certain irreducible homology 3 -spheres, each of which is obtained from two disjoint knot complements $X$ and $Y$ by cross-matching meridians and longitudes. That is, one pastes $\mathrm{bd}(X)$ to $\mathrm{bd}(Y)$ with an orientation reversing homeomorphism so that a meridian of $\mathrm{bd}(X)$ is matched to a longitude of $\mathrm{bd}(Y)$ and a longitude of $\mathrm{bd}(X)$ is matched to a meridian of $\mathrm{bd}(Y)$. W. Browder has suggested to the author that infinitely many of these homology 3-spheres might be topologically distinct. The purpose of this paper is to verify Browder's suggestion.

1. Preliminaries. Let $T$ be a solid torus (of genus one) imbedded in a 3-sphere $S$. A simple loop in bd $(T)$ which bounds a disk in $T$ but not in $\mathrm{bd}(T)$ is called a meridian. A simple loop in $\operatorname{bd}(T)$ which does not link the core of $T$ and which does not bound a disk in $\operatorname{bd}(T)$ is called a longitude. Equivalently, a longitude is a loop on $T$ which is the boundary of a compact surface in $\operatorname{cl}(S-T)$ and which does not bound a disk in $\mathrm{bd}(T)$. Evidently, one may discuss meridians and longitudes not only in 3-spheres but also in knot complements.

A 3-manifold $M$ is called irreducible if every imbedded 2-sphere in $M$ bounds a 3-cell.

LEMMA 1. Let $M$ be the closed 3-manifold which is obtained by crossmatching the meridians and longitudes of two disjoint knot complements $X$ and $Y$. Then $M$ is an irreducible homology 3-sphere.

Proof. Since the meridian of a knot complement generates its homology and the longitude is homologically trivial, a Mayer-

Presented to the Society, March 27, 1970; received by the editors August 12, 1969.

A MS Subject Classifications. Primary 5560; Secondary 5520.

Key Words and Phrases. Homology sphere, torus knot, fundamental group, presentation, symmetric group, representation. 
Vietoris sequence shows that $M$ is a homology 3 -sphere. The corollary to Theorem 1 of the author [1] shows that $M$ is irreducible.

A knot $k$ which lies on the boundary of an unknotted solid torus $T$ in a 3-sphere is called a torus knot. Let $p$ be the number of times (algebraically) that the knot $k$ intersects a meridian on bd(T), and let $q$ be the number of times (algebraically) that the knot $k$ intersects a longitude on $\operatorname{bd}(T)$. There $p$ and $q$ are relatively prime, since otherwise $k$ would not be connected. Let $X$ be the closed complement of the knot $k$. Then the fundamental group of $X$ has the presentation $\left(a, b: a^{p}=b^{a}\right.$ ), where the element $a$ corresponds to the longitude of $\operatorname{bd}(T)$ and the element $b$ corresponds to the meridian of $\operatorname{bd}(T)$. If $n p+m q=1$, then each meridian of $X$ is in the homotopy class of $b^{n} a^{m}$ and each longitude of $X$ is in the homotopy class of $a^{p}\left(b^{n} a^{m}\right)^{-p q}$.

The torus knot $k$ of the preceding paragraph is said to have type $(p, q)$.

2. Homology 3-spheres obtained from torus knots. Let $M_{n}$ denote the irreducible homology 3 -sphere obtained by cross-matching the meridians and longitudes of two disjoint copies of the complement of the torus knot of type $(2,2 n+1)$. By the van Kampen theorem, the fundamental group $\pi_{1}\left(M_{n}\right)$ has the presentation

$$
\begin{aligned}
{\left[a, b, c, d: a^{2}=b^{2 n+1}, c^{2}=d^{2 n+1}, a^{2}\left(a^{-1} b^{n}\right)^{4 n+2}=\right.} & d^{-n} c, \\
& \left.c^{2}\left(c^{-1} d^{n}\right)^{4 n+2}=b^{-n} a\right] .
\end{aligned}
$$

The relations of this presentation for $\pi_{1}\left(M_{n}\right)$ will be designated by the lower case Roman numerals (i), (ii), (iii), and (iv), respectively.

THEOREM. Let $p$ and $q$ be positive integers such that $(4 p+3) !<2 q+1$ and that $2 p+1$ and $2 q+1$ are prime. Then the homology 3-spheres $M_{p}$ and $M_{Q}$ are topologically distinct.

Proof. Lemmas 2, 3, and 4 below show that whereas $\pi_{1}\left(M_{p}\right)$ has a nontrivial representation in the symmetric group $S_{4 p+3}, \pi_{1}\left(M_{q}\right)$ has only trivial representations in $S_{4 p+3}$.

In what follows, if we are considering a representation of $\pi_{1}\left(M_{n}\right)$ into some symmetric group, the notation $x \rightarrow(r, \cdots, s)$ means that the element $x$ of $\pi_{1}\left(M_{n}\right)$ is mapped onto the permutation $(r, \cdots, s)$.

LEMma 2. There is a representation of the group $\pi_{1}\left(M_{n}\right)$ into the symmetric group $S_{4 n+3}$ which maps some element of $\pi_{1}\left(M_{n}\right)$ onto a permutation of order $4 n+3$.

Proof. Consider a function (into $S_{4 n+3}$ ) on the generators of the given presentation for $\pi_{1}\left(M_{n}\right)$ such that $a$ and $c$ go onto the permuta- 
tion $(2 n+1,2 n+2)(4 n+2,4 n+3)$ and $b$ and $d$ go onto the permutation $(1, \cdots, 2 n+1)(2 n+2, \cdots, 4 n+2)$. Relations (i) and (ii) of the presentation are obviously satisfied. The $n$th power of $(1, \cdots, 2 n+1)(2 n+2, \cdots, 4 n+2)$ is the product of a cycle on the letters $1, \cdots, 2 n+1$ and a cycle on the letters $2 n+2, \cdots, 4 n+2$. Thus, the product of $(2 n+1,2 n+2)(4 n+2,4 n+3)$ and the $n$th power of $(1, \cdots, 2 n+1)(2 n+2, \cdots, 4 n+2)$ is a cycle on the letters $1, \cdots, 4 n+3$. Therefore, relations (iii) and (iv) of the presentations are satisfied. Consequently, the function on the generators $a, b, c$, and $d$ extends to a representation.

Lemma 3. Any representation of $\pi_{1}\left(M_{n}\right)$ such that $a \rightarrow 1, b \rightarrow 1, c \rightarrow 1$, or $d \rightarrow 1$ (where $a, b, c$, and $d$ are the generators of the given presentation for $\left.\pi_{1}\left(M_{n}\right)\right)$ takes every element of $\pi_{1}\left(M_{n}\right)$ onto the identity.

Proof. First assume that $a \rightarrow 1$. Then $b^{2 n+1} \rightarrow 1$, by relation (i), and therefore, $\left(b^{n}\right)^{4 n+2} \rightarrow 1$. Since relation (iii) now implies that $d^{-n} c \rightarrow 1$ and, according to relation (ii), $d^{-2 n-1} c^{2} \rightarrow 1$, it is clear that $d \rightarrow 1$ and $c \rightarrow 1$. Relation (iv) now implies that $b^{-n} \rightarrow 1$ and, since relation (i) yields $b^{-2 n-1} \rightarrow 1$, it follows that $b \rightarrow 1$. It is clear from the symmetry of the presentation that if one assumes that $c \rightarrow 1$, it will follow that $b \rightarrow 1, a \rightarrow 1$, and $d \rightarrow 1$.

Next assume that $b \rightarrow 1$. Then $a^{2} \rightarrow 1$, by relation (i), and therefore $a^{-4 n} \rightarrow 1$. Relation (iii) now implies that $d^{-n} c \rightarrow 1$. Since $d^{-2 n-1} c^{2} \rightarrow 1$, it follows that $d \rightarrow 1$ and $c \rightarrow 1$. By relation (iv), $a \rightarrow 1$. By symmetry of the presentation, the assumption $d \rightarrow 1$ also implies that the other generators are mapped onto the identity. Thus, Lemma 3 is proved.

Lemma 4. Let $r$ and $n$ be positive integers such that $(4 r+3) !<2 n+1$ and $2 n+1$ is prime. Then any representation of $\pi_{1}\left(M_{n}\right)$ into the symmetric group $S_{4 r+3}$ is trivial.

Proof. Suppose that the order of the element of $S_{4 r+3}$ which represents the generator $b$ is $m$. Since $m \leqq(4 r+3) !<2 n+1$ and $2 n+1$ is prime, there exist integers $k$ and $t$ such that $(2 n+1) k+m t=n$. Then

$$
\begin{aligned}
d^{-n} c & =(\text { by relation (iii) }) a^{2}\left(a^{-1} b^{n}\right)^{4 n+2} \\
& =a^{2}\left(a^{-1} b^{(2 n+1) k} b^{m t}\right)^{4 n+2}
\end{aligned}
$$

is represented by the same permutation as the element (since $b^{m t} \rightarrow 1$ )

$$
\begin{aligned}
& \left.a^{2}\left(a^{-1} b^{(2 n+1) k}\right)^{4 n+2}, \quad \text { which equals (since } a^{2}=b^{2 n+1}\right) \\
& a^{2}\left(a^{2 k-1}\right)^{4 n+2}=a^{4((2 n+1) k-1)}=a^{4(-m t)}=b^{m(2 n+1)(-2 t)},
\end{aligned}
$$

which is represented by the identity, that is, $d^{-n} c \rightarrow 1$. By relation 
(ii), $d^{-2 n-1} c^{2} \rightarrow 1$. Therefore $d \rightarrow 1$. By Lemma 3 , the representation is trivial.

The proof of the theorem is now complete.

REMARK. Seifert [2] has constructed an infinite class of homology 3 -spheres by doing surgery on torus knots. He shows that the members of this class are distinct by using his theory of fibre spaces, which is more complicated than the methods employed here. Proof that Seifert's examples are irreducible follows from Satz (7.1) and Lemma (10.2) of Waldhausen [3].

\section{REFERENCES}

1. J. L. Gross, Prime 3-manifolds and the doubling operation, (to appear).

2. H. Seifert, Topologie dreidimensionaler gefaserter Räume, Acta Math. 60 (1932), 147-238.

3. F. Waldhausen, Eine Klasse von 3-dimensionalen Mannigfaltigkeiten. II, Invent. Math. 4 (1967), 87-117.

Princeton University, Princeton, New Jersey 08540 and

Columbia University, New York. New York 10027 